\title{
Experience of collaboration between a Dutch surgical team in a Ghanaian Orthopaedic Teaching Hospital.
}

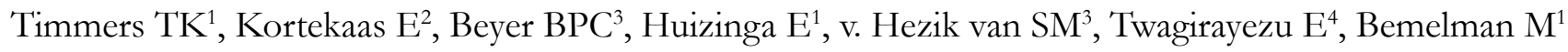

1. University Medical Center Utrecht, Department of Surgery/Trauma-surgery.

P.O.-box 85500, 3508 GA Utrecht, The Netherlands

2. University Medical Center Utrecht, Department of Anaesthesiology

3. University Medical Center Utrecht, Department of Vital Functions and Theatre Managment

4. St. Joseph's Hospital, Department of Orthopaedic Surgery Effiduase-Koforidua, Ghana

\begin{abstract}
Background: Surgery is an indivisible, indispensable part of healthcare. In Africa, surgery may be thought of as the neglected stepchild of global public health. We describe our experience over a 3-year period of intensive collaboration between specialized teams from a Dutch hospital and local teams of an orthopaedic hospital in Effiduase-Koforidua, Ghana.

Intervention: During 2010-2012, medical teams from our hospital were deployed to St. Joseph's Hospital. These teams were completely self-supporting. They were encouraged to work together with the local-staff. Apart from clinical work, effort was also spent on education/ teaching operation techniques/ regional anaesthesia techniques/scrubbing techniques/ and principles around sterility.

Results: Knowledge and quality of care has improved. Nevertheless, the overall level of quality of care still lags behind compared to what we see in the Western world. This is mainly due to financial constraints; restricting the capacity to purchase good equipment, maintaining it, and providing regular education.

Conclusion: The relief provided by institutions like Care-to-Move is very valuable and essential to improve the level of healthcare. The hospital has evolved to such a high level that general European teams have become redundant. Focused and dedicated teams should be the next step of support within the nearby future.
\end{abstract}

Keywords: Orthopaedic, improvement, Ghana, collaboration, Dutch.

DOI: http://dx.doi.org/10.4314/ahs.v16i3.26

Cite as: Timmers TK, Kortekaas E, Beyer BPC, Huizinga E, v. Hezik van SM, Twagirayezu E, Bemelman M. Experience of collaboration between a Dutch surgical team in a Ghanaian Orthopaedic Teaching Hospital. Afri Health Sci 2016;16(3): 838-844. DOI: http:/ / dx.doi. org/10.4314/abs.v16i3.26

\section{Introduction}

Many countries in the developing world face a significant challenge in providing effective primary healthcare ${ }^{1,2}$. Surgery is an indivisible, indispensable part of healthcare $^{3,4,5,6,7}$. In Africa, surgery may be thought of as the neglected stepchild of global public health. There are fewer physicians per population on this continent than on any other; surgeons are $\operatorname{rarer}^{2,8}$. In the last 20 to 30 years African medical treatment, education, and training have

\section{Corresponding author:}

Timmers T.K, University Medical Center Utrecht, Department of Surgery/Trauma-surgery.

P.O.-box 85500, 3508 GA Utrecht, The Netherlands Email: tk.timmers@gmail.com made improvement. However, Africa's health workforce crisis has been emphasized by international organisations; it has become apparent that the workforce required to deliver surgical services has been significant neglected ${ }^{7,9,10,11}$. Therefore it is of great importance that healthcare continues to improve.

This is partially achieved by the intensive collaboration between specialized teams from Western countries (United States/ Europe/ Australia) and the local African hospital teams ${ }^{1,6,8,12}$. Such collaboration exists between various Dutch hospitals and St. Joseph's Hospital in Effiduase-Koforidua. This hospital has dedicated itself to orthopaedic and trauma healthcare and has established itself as a centre of excellence in Ghana, Togo, and Ivory Coast. The high number of motor vehicle accidents, pedestrian injuries, and orthopaedic abnormalities presents 
a heavy workload for the local hospitals, with often very challenging pathology. With the aid of Care-to-Move (a non government charitable foundation), multiple teams from Holland (with orthopaedic and trauma expertise) visit this hospital, several times each year, to improve the level of healthcare. We describe our three year experience of this collaboration in Ghana.

\section{Background}

\section{Care-To-Move}

The foundation Care-to-Move was founded in April 2005. Based on charitable principles, the organisation aims to provide exchange of knowledge and skills concerning trauma and orthopaedic healthcare. The secondary goal is to develop St. Joseph's Hospital into a self-supporting teaching hospital for orthopaedic and trauma-surgical pathology for the Eastern region of Ghana. This exchange involves professional, medical, and organisational expertise from 9 Dutch hospitals deployed primarily to St. Joseph's Hospital in Effiduase-Koforidua. These teams (which mainly consist of trauma-orthopaedic surgeons, anaesthesiologists, and nurses) are committed to improving the quality of care through education, management advice, and performing surgical interventions together with Ghanaian physicians/ surgeons.

\section{St. Joseph's Hospital}

St. Joseph's Hospital was built in the 1950's by the Brothers of St. John of God. The hospital is located in the Eastern region of Ghana (a 2 hour drive from the capital Accra) and serves as a regional referral centre for a population of about 2 million people. They also receive patients from Togo and the Ivory Coast. The main focus is orthopaedic pathology and trauma related injuries. St. Joseph Hospital has been appointed by the Ghanaian Minister of Health for a postgraduate course for orthopaedic and general surgery. The hospital works closely with the central government hospital in Koforidua. Both hospitals participate in a rotational program for medical doctors. The hospital now contains 150 beds, 4 theatre rooms, a plaster room, radiology department, pharmacy, laboratory, blood bank, an outpatient clinic, and an emergency department. The wards and theatre rooms have gradually been relocated in a new building since 2010 .

\section{Dutch Surgical Team}

During the last 3 years our hospital, University Medi- cal Centre Utrecht (UMCU), has provided a team with a trauma-surgeon, an orthopaedic surgeon, an anaesthesiologist, a surgical resident, first aid nurses, and scrubnurses to St. Joseph's Hospital for a period of 14 days per year.

The UMCU serves as a regional Level 1 trauma centre and a regional referral centre with 1042 beds. The emergency department treats 30000 patients each year, including 300-400 poly-trauma patients. The trauma and orthopaedic department have extensive experience in primary and secondary fracture and orthopedic care. As a teaching hospital the UMCU has a close relation with the Arbeitsgemeinschaft für Osteosynthesefragen (AO) foundation ("Association for the Study of Internal Fixation"). The AO-foundation focuses on new principles and techniques related with trauma and orthopaedic surgery. This makes them a valuable partner for international clinical and educational networking among orthopaedic and trauma professionals ${ }^{13}$.

Patients' informed consent has been given for publication of the photos as educational imaging.

\section{Experience}

During 2010 - 2012 different medical teams were deployed to St. Joseph's Hospital. These teams were completely self-supporting. However, they were encouraged to work together with the local staff. Main indications for treatment were fractures, malunions, non-unions, infections (osteomyelitis), clubfoot, and other congenital bone abnormalities. Apart from the clinical work much effort was spent on education, operation techniques, regional anaesthesia techniques, scrubbing techniques, and principles around sterility.

\section{Education}

\section{Anaesthesia:}

Due to a limited recovery capacity and facilities, regional techniques were mainly used for anaesthesia in St. Joseph's Hospital. Most anaesthesia is performed by well trained and enthusiastic anaesthesia nurses. They have a vast experience with spinal anaesthesia. Nevertheless, they were in need of education on this topic; especially education concerning loco-regional anaesthesia with a nerve stimulator (skills).

Initially three theoretical sessions were given in which they were trained to carry out the following nerve blocks: the scalanus, brachial, ischial, femoral, and popliteal. Emphasis was placed on the anatomical landmarks and the

African Health Sciences Vol 16 Issue 3, September 2016 
use of a nerve stimulator. Posters were made showing all landmarks and safe zones to introduce the needles. After that, the nurses gradually started performing these procedures on patients under supervision of our anaesthesiologist. The goal was to perform this technique safely, respecting sterility, and checking meticulously for intravascular routes. Due to the basic skills of the local nurses and their enthusiasm we were able to teach these techniques within 2 weeks. The postoperative advantages, with prolonged pain reduction, proved to be a huge benefit for the patient care on the ward. Due to the success and the higher level of patient care, this technique was adapted in their system. A regular supply of local anaesthetics and needles were put on the supply list. The nerve stimulator with extra batteries was donated to the hospital.

\section{Operating room (OR) personnel:}

The OR personnel intervened on several fields within the operating room. First, we noticed a lack of interaction between the OR-nurses and the circulating nurses. In order to perform an adequate and safe operation it is of great importance to foresee the needs of the surgeon and the OR-nurse, and to prepare the instruments pro-actively. Therefore, we decided that the visiting OR-nurse would be scrubbing in whilst orchestrating the input of the local circulating nurses on the first week. In the second week they switched places. Due to this method, we were able to improve timing, plan procedures more efficiently, and increase the knowledge of the operative technical aspects.

Secondly, we also made an effort to change the awareness of sterility in and around the theater (complex). Education was given on how to work and handle a sterile environment. Although preoperative cleaning and sterilization of tools was professionally executed, we recognized several shortcomings concerning sterility during surgery. For example: the batch of sterilized sets was used throughout the whole day, each item being lifted out of the tray with a "sterile" clamp. We changed the routine in which more specific sets for each operation were prepared and that these sets were returned directly after the procedure in order to be re-sterilized completely.

\section{Organisation}

Before 2011, St. Joseph's Hospital did not have a selforganising and supporting emergency department. Emergency patients were brought to the outdoor patient department and were treated by the physician who was doing outdoor patient management, when he had the time. Besides the possibility of doing $\mathrm{x}$-ray investigation, there was no facility to perform an emergency treatment/ resuscitation properly. In 2011, our team created, an effective but easy to run, self-supporting emergency room facility. With simple structural improvements: e.g. Dedicated rooms, a "Red ", "Yellow", and "Green" bay; Simple treatment tables and basic equipment like IV needles and BP measuring equipment. Local outdoor patient nurses were trained in emergency procedures and structural emergency organisation.

Concerning the in-hospital patient-management, each day a morning report was introduced to discuss newly admitted/ operated patients. The report was attended by the medical director and all available specialists.

Every Tuesday, with the help of other teams as well, a grand round was gradually introduced. The surgical/ orthopaedic staff, physiotherapist, attending nurse, orthopedics plaster of Paris (POP) master, and our medical team attended this round. This proved to be a valuable moment to discuss past results, planned operations, and provide transparency. In the afternoon there was a preoperative small round in which the anaesthesiologist together with the surgeon and resident checked all patients planned for operation for the next day.

\section{Patients and surgical procedures}

When arriving in 2011, 99 patients were already admitted and waited for a surgical intervention. We were able to treat (operate) 37 of these patients: 23 patients had fractures which needed stabilisation, 9 patients were operated because of a longstanding soft tissue or bone infection (osteomyelitis), 2 patients underwent surgical reconstruction for their congenital bone abnormality, and 3 patients were treated for other pathologies. During our last visit, 2012, 66 patients were already admitted and in need of a surgical intervention.

Fifty-two patients received an operation: 33 of these patients had fractures which needed stabilisation [Fig 1], 6 patients were operated because of a longstanding soft tissue or bone infection [Fig 2], 5 patients underwent a surgical reconstruction for their congenital bone abnormality [Fig 3], and 8 patients were treated for other pathologies.

With closed fractures we were able to perform an open reposition with internal fixation (ORIF) with the current 
Figure 1:

One of several patients with a compound open (Gustilo grade 2) cruris (lower leg) fracture (AO.42.C2) which we treated.

A: Picture of the open (Gustilo grade 2) cruris (lower leg) fracture (AO.42.C2).

B: Lower leg x-ray revealing the cruris (lower leg) fracture (AO.42.C2).

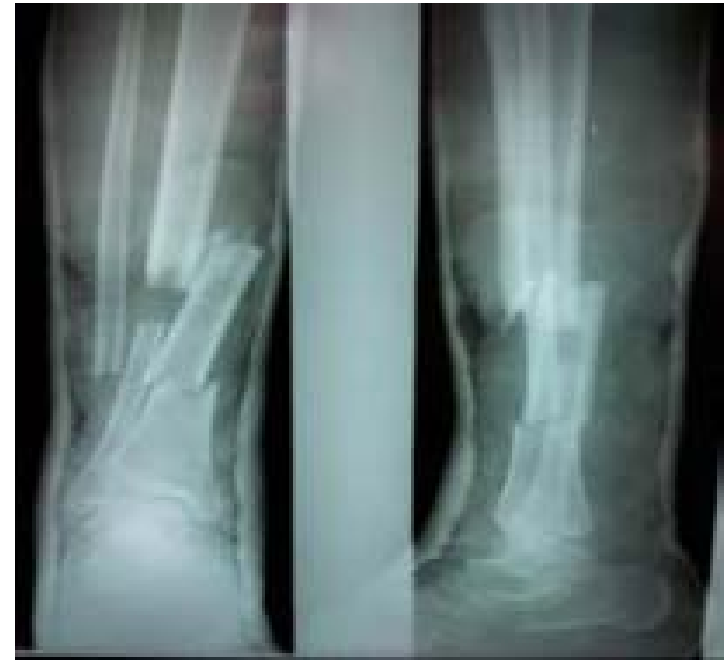

$1 \mathbf{A}$

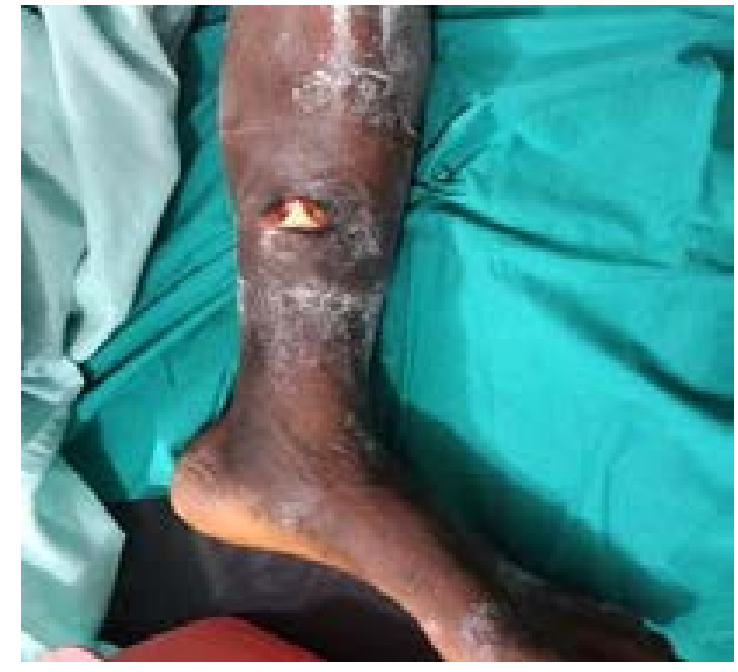

$1 \mathbf{B}$

This injury was several weeks old, treated with a plaster and antibiotics until surgery. We removed the sequester (sticking out of the wound) and stabilized the fracture with a dynamic compression plate. We needed to remove a couple of $\mathrm{cm}$ of the fibula because the fracture was several weeks old and, therefore, we were not able to get enough length.

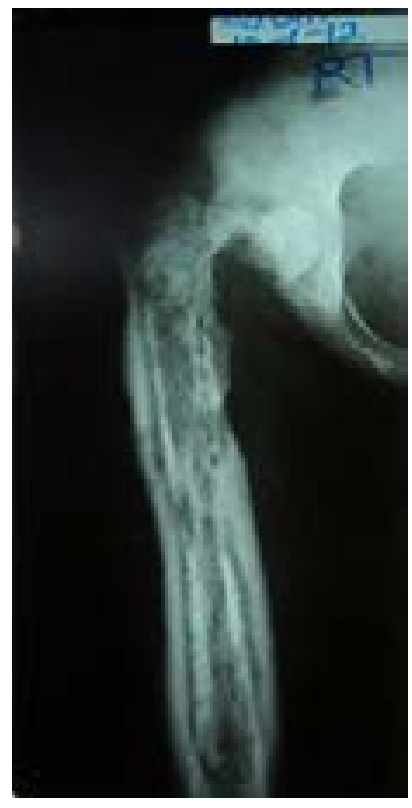

2A

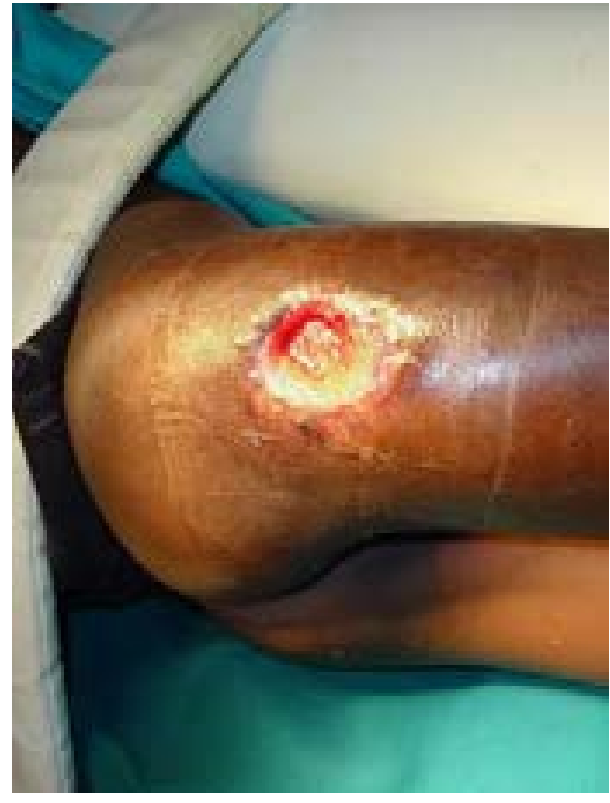

2B

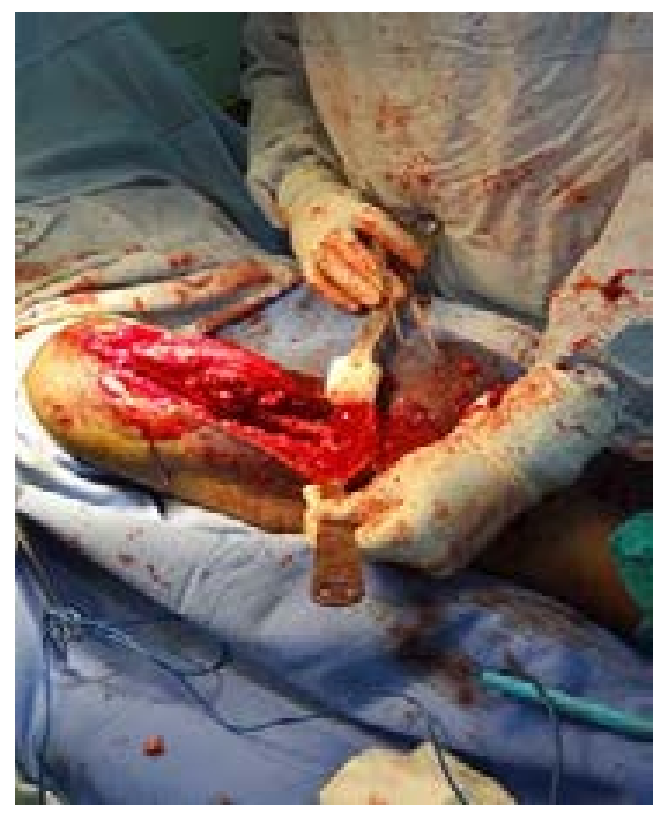

2C

Figure 2:

One of the patients with osteomyelitis (femur).

A: Picture of the entree ulcus at the level of the right hip (greater trochanter).

B: Femur x-ray showing a very extensive osteomyelitis of the complete femur shaft.

C: Perioperative picture of the surgical approach for the osteomyelitis debridement. 


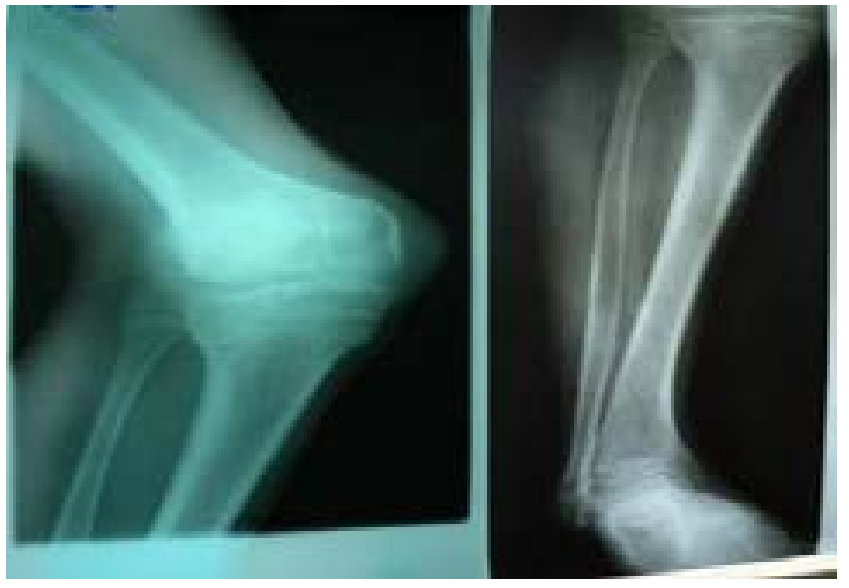

3A

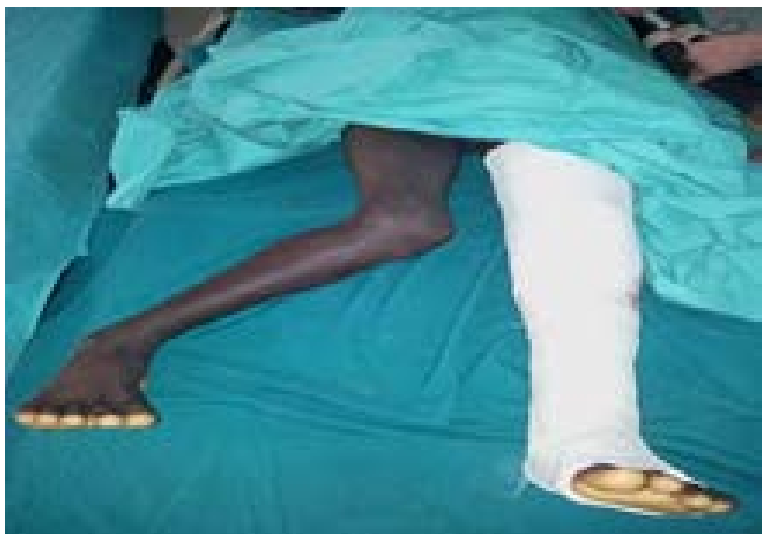

3C

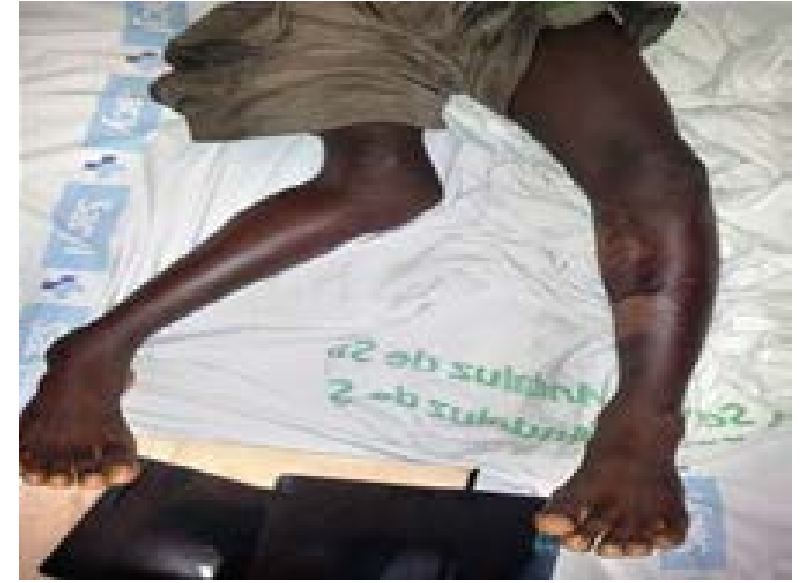

3B

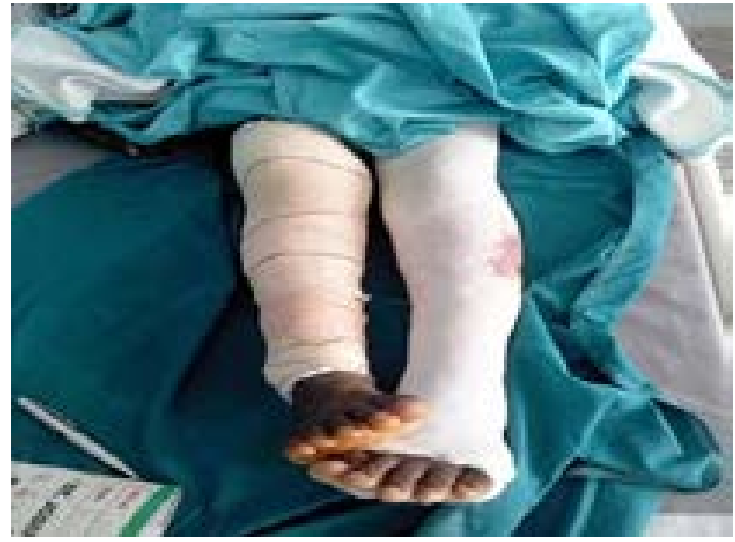

3D

\section{Figure 3:}

Congenital bone abnormality: this patient had a varus deformity of the left knee, and a valgus deviation of the right knee with also a rotational deformity of the femur.

A: Pre-operative situation.

B: X-rays of both legs before the operative correction.

C: Post-operative situation after the first correction (angled osteotomy of the tibia at the left side to correct the varus deformity).

D: Post-operative situation after the second intervention (angled osteotomy of the Femur with a corresponding derotation angled tomy of the proximal tibia and an angled osteotomy of the distal part of the tibia).

techniques: bridging or compression plate fixation. A favourite and strong instrument is the blade-plate; a somewhat 'old fashioned' instrument but of great value in the African setting. A considerable delay, unfortunately, was customary with most patients; therefore,anatomical reduction was difficult or even impossible. This mandated a strong implant which is provided with the blade plate.

The open (mainly grade 2 and 3 ) fractures, were treated mainly with a massive debridement and external fixator. This was also due to a long delay prior to presentation, resulting in severe contaminated or infected wounds. Simi- lar selected cases in Europe, with such a severe infection, would receive an amputation. However, in Africa this is a different ball game. The rehabilitation capabilities are very poor together with the social impact in becoming an amputee (and almost automatically an outcast of society) made us realize that amputation in Africa is only an option to prevent death. And even then, we had to accept that the social pressure can be so high that some patients preferred death to an amputation.

A frequently seen and almost eradicated pathology in the Western world is the primary osteomyelitis. This was 
mainly seen in children and adolescents. In the majority of these cases a huge and well distinguishable sequester could be found on the conventional x-rays [Fig. 2B]. All patients underwent a complete debridement with removal of sequesters through an osteotomy. In select cases the sequesters were so extensive that we had to be careful not to remove more that $1 / 3$ of the circumference of the bone, and therefore preventing an iatrogenic fracture. Postoperatively, IV antibiotics were given to treat the infection. Often a double therapy was given consisting of a cephalosporin and an aminoglycoside. In this aspect, our team respected the local knowledge concerning the often very aggressive infections and their antibiotic regimes.

\section{Discussion}

The condition in which patients arrive at the hospital in Koforidua is completely different from what we are used to in Europe. The pre-hospital management in Ghana is virtually non-existent. This results in a significant prolonged presentation at the outpatient clinic or emergency department. Furthermore, patients have often already been "treated" by an herbal doctor. Unfortunately, this did not improve the local condition of the injury and transformed an initial "simple" problem into the most challenging pathology with secondary necrotic tissue and/ or infection in most cases.

Education has been improved and increasingly been given in the last decade. A fast improvement of the knowledge concerning orthopaedic and trauma principles in St. John of God Hospital in Koforidua is seen. Nevertheless, the overall level of quality of care still lags behind compared to what we see in the Western world ${ }^{1,10-12}$. This is mainly due to financial constraints with the result of restricting the capacity to purchase good equipment, deteriorating maintenance, and moderate and unreliable education. Unfortunately, many surgeons in Ghana have been trained quite a while ago and they have not had the opportunity to keep their knowledge up-to-date. This resulted in the use of outdated techniques. As mentioned above, the overall level of knowledge is lower than the Western world. However,this does not account for the whole healthcare system, for example the POP techniques. These techniques originate from half a decennium ago. Nevertheless, it proves to be extremely valuable in countries like Ghana. The techniques to treat open book injuries of the pelvis with hammock traction are almost completely forgotten and in fact obsolete in the Western world. Seeing these techniques used with great profession and pride is a learning moment for the modern teams from Europe.

Realizing where healthcare was 10 years ago and where it is now, confirms that the relief provided by institutions like Care-to-Move are very valuable and essential to improve the level of healthcare $1,6,8,12$. The foundation Careto-Move has been involved in improving the St. Joseph's orthopaedic Hospital in Effiduase-Koforidua, Ghana, since 2005. Through their support (sponsoring) and the Ghanaian government itself, a new hospital has been realized and healthcare improved considerable. Not only the pure clinical knowledge, but also the organisation behind it, needed improvement (providing a maintenance programme, adequate supply management, financial management, etc). At this moment, the hospital has currently evolved to such a high level that general European teams have become redundant. The time has come to change the help in small, very focused, and dedicated teams.

\section{Conclusion}

Collaboration between Western world medical teams and local African teams is considered to be of great importance for both parties. When adjusting healthcare in Africa, not only the pure clinical knowledge needs to be improved, but also the organisational structure of a hospital needs to be addressed. This structure has to be rearranged throughout all healthcare layers within a hospital society. Such a change is only possible with the support of the (local) government and the endless effort of charitable foundations like Care-to-Move. However, when the time is right, such collaboration should change for the benefit of the local healthcare system.

Points for the future would be to introduce procedures concerning hemi and total hip prosthesis. The amount of patients with destroyed hip joints is tremendous. Till now, the only treatment was a Girdlestone procedure. Providing prosthesiology would be a great benefit for all patients with hip-pathology. Embarking this next goal mandates more strict principles concerning sterility, operative technique, and post-operative management.

Other special concerns for the future must be directed that the knowledge of fracture management does not de-

African Health Sciences Vol 16 Issue 3, September 2016 
crease and stays up-to-date. In order to ensure this, the current orthopaedic staff should participate regularly in education programs like the $\mathrm{AO}^{13}$.

\section{Acknowledgments and funding}

We received no source of funding for our study.

\section{Conflict of interest}

The authors declared that they have no competing interests.

Each author certifies that he or she has participated sufficiently in the intellectual content, the analysis of data, if applicable, and the writing of the work to take public responsibility for it. Each has reviewed the final version of the work, believes it represents valid work, and approves it for publication. We herewith state that all authors provided an equal contribution to this study and act in concordance to the 'Uniform Requirements'.

Timmers carried out the complete acquisition of data, carried out the full analysis and interpretation of data, and made the draft of the manuscript. Bemelman carried out a substantial draft of the manuscript, had supervision of the study design, and had done critical revisions to the manuscript for important intellectual content. Kortekaas, Beyer, Huizinga, van Hezik, and Twagirayezu participated in the concept and design of the study, helped in the acquisition of data, performed the analysis and interpretation of data.

\section{References}

1. Wright IG, Walker IA, yacoubmh. Specialist surgery in the developing world: luxury or necessity? Anaesthesia 2007; 62: 84-89.

2. Alkire BC, Raykar MG, Weiser TG, et al. Global access to surgical care: a moddeling study. Lancet Glob Health
2015; 3: E316-323. Doi: 10.1016/s2214-109x(15)70115-4. 3. Meara JG, Greenberg SL. Global surgery as an equal partner in health: no longer the neglected stepchild. Lancet Glob Health 2015; 3: S1-2. Doi: 10.1016/S2214109X(15)70019-7.

4. Brugha R, Bijlmakers L, Borgstein E, Kachimba J, COST-Africa Consortium. The evidence needed to make surgery a global health priority. Lancet Glob Health 2015; 3: E741. Doi: 10.1016/S2214-109X(15)00181-3.

5. Wall E. Ethics in global surgery. World J Surg 2014; 38: 1574-1580. Doi: 10.1007/s00268-014-2600-5.

6. Ologunde R, Maruthappu M, Shanmugarajah K, Shalhoub J. Surgical care in low and middle-income countries: burden and barriers. Int J Surg 2014; 12: 858-863. Doi 10.1016/j.ijsu.2014.07.009.

7. Kingham TP, Kamara TB, Cherian MN, et al. Quantifying surgical capacity in Sierra Leone: a guide for improving surgical care. Arch Surg 2009; 144: 122-127 pubmed . Doi: 10.1001 /archsurg.2008.540.

8. Farmer PE, Kim JY. Surgery and Global Health: A View from Beyond the OR. World J Surg 2009; 32: 533536. Doi: 10.1007/s00268-008-9525-9.

9. Ozgediz D, Galukande M, Mabweijano J, et al. The neglect of the global surgical workforce: experience and evidence from Uganda. World J Surg 2008; 32: 1208-1215. Doi: 10.1007/s00268-008-9473-4.

10. Notrica MR, Evans FM, Knowlton LM, Kelly mcqueen KA. Rwandan surgical and anesthesia infrastructure: a survey of district hospitals. World J Surg 2011; 35: 1770-1780. Doi: 10.1007/s00268-011-1125-4.

11. Lavy C, Sauven K, Mkandawire N, et al. State of surgery in tropical Africa: a review. World J Surg 2011; 35: 262-271. Doi: 10.1007/s00268-010-0885-6.

12.Wolfberg AJ. Volunteering overseas - lessons from surgical brigades. N Engl J Med 2006; 354: 443-445

13.Https://www.aofoundation.org/Structure/Pages/default.aspx 